\title{
COMPORTAMIENTO DINÁMICO DE UNA EMBARCACIÓN PESQUERA EN EL MAR PERUANO
}

\section{COMPORTAMIENTO DINÁMICO DE UNA EMBARCACIÓN PESQUERA EN EL MAR PERUANO}

\author{
Hugo Eliseo Gamarra Chinchay ${ }^{1}$, Anwar Julio Yarin Achachagua ${ }^{2}$, Yasser Hipólito Yarin \\ Achachagua $^{3}$, Mierwen Palacios Aranda ${ }^{4}$
}

\begin{abstract}
RESUMEN
El presente trabajo describe la metodología utilizada para predecir el comportamiento presentado por una embarcación de desplazamiento cuando ésta navega en determinada condición de mar. Es importante que el ingeniero naval determine este comportamiento en la fase de proyecto de la embarcación, porque de esta forma puede evaluar los efectos perjudiciales para su desempeño, como por ejemplo la emersión del propulsor, embarque de agua en la cubierta, slamming y excesivas aceleraciones verticales. Los efectos perjudiciales descritos anteriormente son predichos a través de los espectros de respuesta de la embarcación para una determinada condición de mar y para un determinado movimiento. El procedimiento utilizado para la obtención de los Espectros de Respuesta consiste en la superposición del Operador de Respuesta Unitaria en olas regulares de la embarcación para un determinado movimiento (RAO) y el Espectro de Energía de Pierson-Moskowitz, utilizado para representar el mar en una determinada condición. El Operador de Respuesta Unitaria de la embarcación fue determinado utilizando un programa de Dinámica de Navíos basado en la Teoría de las Rebanadas (Strip Theory) y; las diversas condiciones de mar consideradas, fueron caracterizadas utilizando datos metereológicos como la altura significativa (Hs) y periodo medio (Tm) de las olas, siendo estos datos importantes en la generación del Espectro de Energía para determinado estado de mar. Así mismo, se muestran los resultados del comportamiento de una embarcación pesquera operando en diversas condiciones típicas del litoral peruano. Los efectos perjudiciales fueron determinados y se evaluó su severidad en el desempeño de la embarcación a través de criterios de diseño.
\end{abstract}

Palabras clave.- Emersión del propulsor, Operador de respuesta unitaria, Espectro de energía de Pierson-Moskowitz.

\begin{abstract}
This paper describes the methodology used to predict the behavior presented by a displacement boat when it sails in certain sea conditions. It is important that the naval engineer determines this behavior in the design phase of the boat, because this way you can evaluate the adverse performance, such as the rise of the propellant, shipping water on deck, slamming and excessive accelerations vertical. The harmful effects described above are predicted by the response spectra of the vessel for a given sea condition and for a given movement. The procedure used for obtaining Response Spectra consists of overlapping Unitary Operator Response in regular waves for a given vessel movement (RAO) and the energy spectrum of Pierson-Moskowitz, used to represent the sea in a certain condition. The operator of the boat united response was determined
\end{abstract}

\footnotetext{
${ }^{1}$ Magister Ing., docente de la Facultad de Ingeniería Mecánica de la Universidad Nacional de Ingeniería, ${ }^{2}$ Egresado de Ingeniería Mecánica de la Universidad Nacional de Ingeniería, ${ }^{3}$ Egresado Facultad de Ingeniería Mecánica de la Universidad Nacional de Ingeniería, ${ }^{4}$ Egresado Facultad de Ingeniería Mecánica de la Universidad Nacional de Ingeniería.
} 
using a Dynamic Vessel program based on the Theory of slices (Strip Theory) and, the various sea conditions considered, were characterized using meteorological data as significant height (Hs) and mean period (Tm) of the waves, these data being important in the generation of the energy spectrum for a given sea state. It also shows the results of the behavior of a fishing vessel operating in various conditions typical of the Peruvian coast. Adverse effects were determined and assessed for severity in the performance of the boat through design.

Keywords.-Emersion propeller, Unitary operator response, Energy spectrum Pierson-Moskowitz.

\section{INTRODUCCIÓN}

El presente estudio, muestra el análisis del comportamiento de un buque pesquero en nuestro mar. Para dicho fin se hará uso de los conceptos de la Dinámica del Buque. Así mismo se empleará el software Ship Motion and Sea Load Computer Program desarrollado por Naval Ship Research and Development Center.

\section{PLANTEAMIENTO DEL PROBLEMA}

En nuestro país la industria de la Construcción Naval juega un rol importante en el diseño y fabricación. Existen astilleros que no realizan un diseño adecuado debido a que no consideran muchos de los efectos que mostraremos a continuación en su diseño.

\section{FUNDAMENTO TEÓRICO}

Programa Ship Motion and Sea Load Computer.Para poder usar este software y entender adecuadamente cada uno de los resultados que este proporciona, es necesario conocer a cabalidad en que teorías está fundamentado este software que fue desarrollado por NSRDC.

Teoría de fajas.- La respuesta de un barco moviéndose en un fluido que a su vez contiene el efecto del oleaje, es un fenómeno muy complejo, pues, contiene la interacción entre la dinámica del barco, la hidrodinámica del fluido, la coexistencia en dos medios muy diferentes, fluido y gas, y el efecto extra del oleaje.

Debido al gran coste de producción de un barco, es interesante poder hacer un estudio teórico del comportamiento de este a priori. Para ello se ha desarrollado toda una teoría, la teoría de franjas o fajas. Esta teoría obtiene los coeficientes de unas ecuaciones lineales que generan un comportamiento muy semejante al comportamiento real del barco.

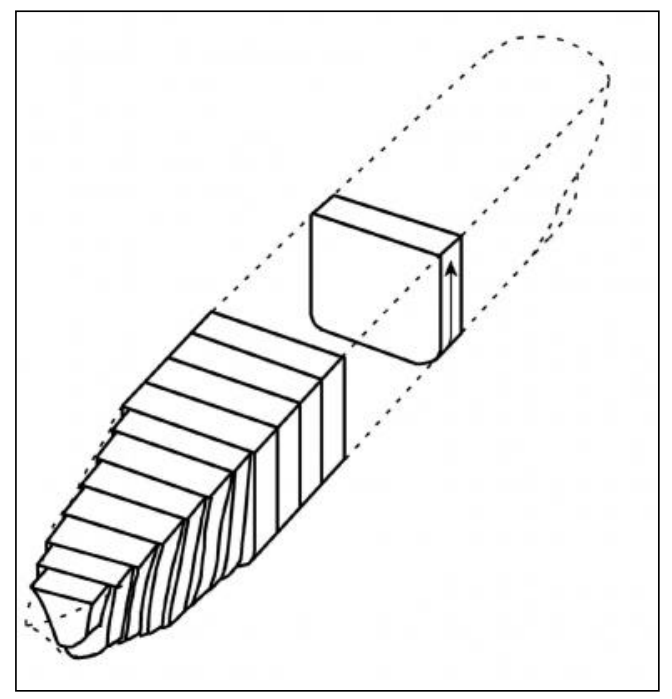

Fig. 1 Casco del buque representado por un número finito de fajas.

Además los cálculos teóricos permiten ampliar el rango de estudio, incluso realizar experimentos que en el barco real o en la maqueta son difíciles de conseguir.

El estudio teórico se ha realizado sobre un oleaje regular y se extrapola a mares irregulares basándonos en que el mar irregular es una composición de olas regulares.

Un barco moviéndose con una velocidad media, sin ningún tipo de sujeción, enfrentándose a un oleaje posee seis grados de libertad, tres de traslación y tres de rotación, como se muestra en la Figura 1. 


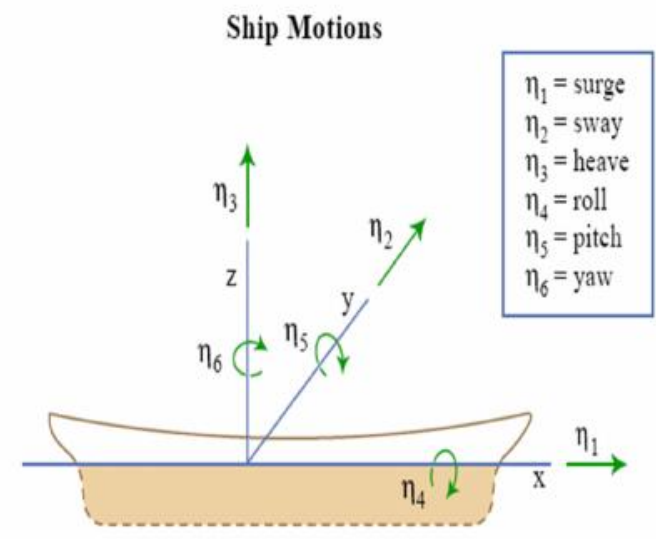

Sign convention for translatory \& angular displacements Figure by MIT OCW.

Fig. 2 Convención de signos para desplazamientos angulares $y$ de translación.

El sistema de referencia que vamos a tomar será el mismo que el del CEHIPAR, para el convenio de signos utilizaremos los ejes de la mano derecha. Para referirnos a los diferentes movimientos utilizaremos tanto la nomenclatura española como la inglesa:

Surge.- Avance del barco, positivo hacia adelante. Normalmente se trabajará con modelos linealizados en torno a una velocidad. Debido a las fluctuaciones en la velocidad, el barco va a estar por delante o por detrás del punto esperado si navegase a velocidad constante. Esta desviación es lo que denominaremos surge.

Sway.- Desplazamiento lateral, lo consideraremos positivo hacia babor, izquierda.

Heave.- Arfada o altura del barco, positivo hacia arriba.

Roll.- Escora o balanceo lateral del barco, positivo es hundir la parte derecha del barco, estribor, rotación respecto a eje $\mathrm{X}$.

Pitch: Cabeceo vertical del barco; positivo hundir la proa, rotación respecto a eje Y.

Yaw.- Guiñada o Cabeceo horizontal del barco; positivo girar hacia la izquierda, rotación respecto a eje $\mathrm{Z}$.

No se contemplarán los grados de flexión del barco por complicar excesivamente el problema y no ser muy importante en el estudio que se quiere realizar. Estos grados de libertad conllevan seis ecuaciones diferenciales no lineales de movimiento, acopladas entre sí. Si se considera un barco simétrico por babor y estribor, entonces las ecuaciones se pueden desacoplar en dos grupos, incluso se pueden aproximar por ecuaciones lineales. Un grupo lo constituye el movimiento longitudinal: surge, heave y pitch. El otro grupo se encarga del movimiento lateral del barco: sway, roll y yaw. Nos quedaremos con las ecuaciones del movimiento longitudinal al ser este el que queremos controlar.

Vamos a utilizar dos sistemas de referencia, uno denominado ejes tierra "Earth" cuyo origen se encuentra fijo en tierra y a la altura de la línea de aguas tranquilas, coincidiendo en dirección la velocidad del barco y el eje $\mathrm{X}$, y otro denominado ejes cuerpo "Body" que se encuentra fijo al centro de gravedad del barco y en continua orientación con él. El ángulo que forman la velocidad de avance del oleaje con respecto a la velocidad del barco, $\mu$, será $180^{\circ}$ cuando el barco se encuentre las olas en sentido contrario.

El comportamiento del buque cuando está en el mar tendrá una relevancia importante con la incidencia de las olas por donde navegara el buque, algunas de estas serán:

- Resistencia estructural.

- Estabilidad.

- Movimiento.

- Velocidad y potencia.

- Incidencia de agua en cubierta.

- Impacto contra las olas.

Cuando las olas inciden con el buque este tiene que responder con una buena resistencia estructural. El buque tiene que tener una buena estabilidad desde su diseño para soportar condiciones críticas cuando entra en contacto con las olas .En cuanto a los movimientos del buque, si estos movimientos son excesivos serán indeseados sobre todo cuando los buques lleven pasajeros, por lo cual se requieren de un confort. En cuanto a la velocidad y la potencia las olas causaran que la resistencia al avance aumente por consiguiente la velocidad de avance del buque disminuye, además producirán condiciones desfavorables para el sistema de propulsión. 
La incidencia de agua en la cubierta dependerá del francobordo y de la altura mínima de proa. El impacto de proa producirá lo que se conoce como SLAMING, que se refiere al impacto de la proa con las olas ,este efecto tendrá importancia relevante de entre $10 \%$ a $25 \%$ de la eslora entre perpendicular .

\section{Incidencia del buque con la ola}

Cuando nos referimos a las olas que se producen en el mar nos referimos a olas que tienen una característica de ola gravitacional regular; esto quiere decir que son olas producidas y formadas por gravedad, mareas altas y mareas bajas. Estas olas están caracterizadas por su periodo (T) y frecuencia (Wo)

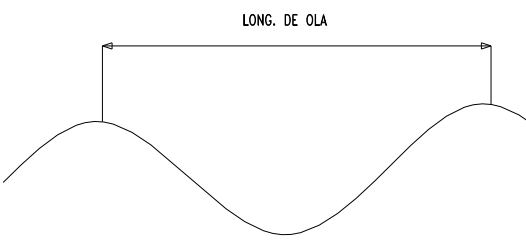

Fig. 3 Periodo (T), frecuencia(Wo), longitud de $\operatorname{ola}(\lambda) \cdot \lambda=\frac{2 \pi g}{W o^{2}}$

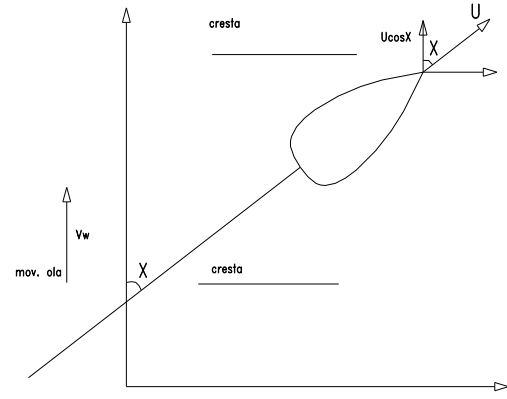

Fig. 4 X ángulo entre la velocidad del buque y la velocidad de la ola.

Del grafico anterior se puede observar que la velocidad relativa entre el buque y la ola esta dado por:

\section{$\mathrm{Vw}-\mathrm{U} \cos \mathrm{X}$}

Por otro lado también el podemos decir que el tiempo requerido para que el buque alcance la próxima cresta (Te). Este tiempo está dado por la siguiente relación básica.

$$
\mathrm{Te}=\frac{\lambda}{V w-U \cos X} \ldots \ldots \ldots \ldots \ldots
$$

También se sabe que :

$$
\mathrm{We}=\frac{2 \pi}{T e}
$$

$\mathrm{Vw}=\frac{\lambda}{T}=\frac{2 \pi g}{W o^{2} T}$

Remplazando (1) en (2)

$\mathrm{We}=\frac{2 \pi(V w-U \cos X)}{V w T}$

Ordenando los términos teniendo en cuenta la relación (3)

$\mathrm{We}=(W o)\left(1-U \frac{W o \cos X}{g}\right)$

La relación (4) nos representa la frecuencia de encuentro del buque con las olas.

En la Figura 5 se observa la consideración principalmente de este estudio.

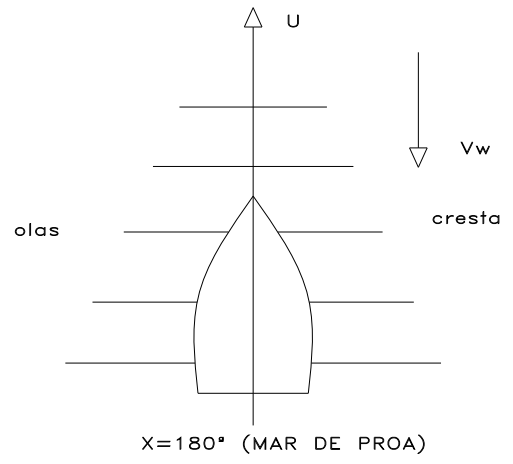

Fig. 5 Consideración de un mar de proa.

\section{Características de las olas en el mar}

La característica más importante de las olas oceánicas es su irregularidad esto en el espacio y en el tiempo .Para los cálculos de seakeeping. El estado del mar mantiene sus características estadísticas, esto es estadísticamente estacionario. 


\section{Seakeeping}

Este efecto se da cuando ocurren en el buque embarque de agua por la proa, golpe de la proa con el mar (SLAMING), efecto de aceleraciones especialmente verticales debido al HEAVE y PITCH,en puntos críticos a lo largo de la eslora del buque, pérdidas de velocidad. Debido a la resistencia adicional existente en olas y debido a reducciones voluntarias, rotación en vació del motor; debido a la inmersión del propulsor

\section{Generación de las olas}

Las olas son generadas por la interacción entre el viento y la superficie del agua, esto a través de:

- Fuerzas de fricción entre los dos fluidos.

- Campo de presión local que cambia en espacio y tiempo.

- Si las olas son de corta amplitud luego la propagación y dispersión de olas es gobernado por el principio de superposición.

- El estado del mar resulta por múltiples interacciones entre el viento y la superficie libre, que varía en espacio y tiempo.

\section{Respuestas verticales}

En términos prácticos la alta frecuencia es un problema como las bajas frecuencias, las fuerzas externas son denominadas por fraude-krilov fuerzas restauradoras.

En cuanto al movimiento de las olas ACRO frecuencias de encuentro, se podrá tener problemas con una alta respuesta irreal.

En general la teoría de franjas da buenos resultados al movimiento vertical del buque.

Referente al movimiento de roll la viscosidad húmeda, es importante, y una buena estimación de este coeficiente es esencial.

La predicción del momento flector vertical y la fuerza cortante son satisfactorias para el gran coeficiente de bloque del buque.

Para coeficiente de bloque pequeño el método es no viable a representar el asimétrico del momento flector vertical.

\section{Respuestas horizontales}

En general uno puede expresar una teoría de franjas pudiendo no ser buenos para las respuestas verticales.

Desde allí son fuerzas no restauradoras la buena estimación de la radiación y difracción de fuerzas todavía para pequeñas frecuencias es importante.

\section{COMENTARIOS}

Este capítulo presenta con frecuencia soluciones para el problema de los movimientos y cargas estructurales inducidas a buques que avanzan en olas armónicas.

La solución está basada en la teoría de franjas que aproxima el medio lineal 3D reduciéndolo a $2 \mathrm{D}$ alrededor de cruce de secciones.

Los efectos del la velocidad a proa son introducidas en un muy simplificado modo y cuenta solo al ángulo de ataque del casco con el flujo hacia el infinito.

Las simplificaciones son:

- El casco es muy delgado el vector normal a la superficie del casco es 2D.

- La frecuencia de oscilación es alta.

- La incidencia de las olas y los movimientos son de pequeñas amplitudes.

\section{ANÁLISIS EN MAR IRREGULAR}

Para empezar en este análisis se debe seleccionar el estado de mar que refleja la cantidad de energía y su distribución entre las olas componentes presentes y que están incidiendo sobre la embarcación

En este caso, se ha considerado realizar el análisis para los estados de mar 2, 3, 4 y 5, cuyas características se muestran en la Tabla1. 
Tabla1.Caracteristicas para el estado del mar.

\begin{tabular}{lllllll}
\hline Tipo de mar & $\mathrm{T}(\mathrm{s})$ & $\begin{array}{l}\mathrm{Lw} \\
(\mathrm{m})\end{array}$ & Hs $(\mathrm{m})$ & Lw/Lpp & Lw/Hs & Hs (pies) \\
\hline 2 & 2.9 & 13.131 & 0.427 & 0.429 & 30.771 & 1.4 \\
3 & 4 & 24.981 & 1.006 & 0.816 & 24.836 & 3.3 \\
4 & 5.1 & 40.610 & 1.859 & 1.327 & 21.842 & 6.1 \\
5 & 5.7 & 50.727 & 2.438 & 1.658 & 20.803 & 8 \\
\hline
\end{tabular}

Donde:

$\mathrm{Lw}=$ Longitud de la Ola

$\mathrm{Hs}=$ Altura significativa de la Ola

$\mathrm{T}=$ Periodo

El análisis desarrollado se realizo siguiendo el criterio de la Tabla 2.

Tabla 2. Criterios de análisis.

\begin{tabular}{ll}
\hline DESPLAZAMIENTO & VELOCIDAD \\
\hline $100 \%$ & 8 nudos \\
401.7 TON & 11 nudos \\
$75 \%$ & 8 nudos \\
301.27 TON & 11 nudos \\
\hline
\end{tabular}

Además se considero que el ángulo de incidencia del buque con las olas será de 180, o igualmente llamada seahead.

Para describirlo analíticamente, se escogió entre varios espectros matemáticos al de la ITTC ec5, el cual está basado en un único parámetro que es la altura significativa de la ola, cuya función básica es determinar el valor de dos constantes A ec6 y B ec7.

$$
\mathrm{S} \eta(\omega)=\left(\mathrm{A} / \omega^{\wedge} 5\right)^{*} \mathrm{EXP}\left(-\mathrm{B} / \omega^{\wedge} 4\right)
$$

Donde Ay B se definen de la siguiente manera:

$$
\begin{aligned}
& \mathrm{A}=8.1^{*} 10^{\wedge}-3^{*} \mathrm{~g}^{\wedge} 2 \\
& \mathrm{~B}=3.11 / \mathrm{H} 1 / 3^{\wedge} 2
\end{aligned}
$$

H1/ 3 = Altura significativa de la Ola $\mathrm{g}=$ aceleración de la gravedad

Como resultado de la aplicación del espectro matemático seleccionado se obtuvo el espectro de mar en función de la frecuencia de olas, para así poder determinar su espectro de Mar modificado en función de la frecuencia de encuentro, cuyas representaciones graficas se muestran en la Figura 6.

Espectro del Mar - ITTC

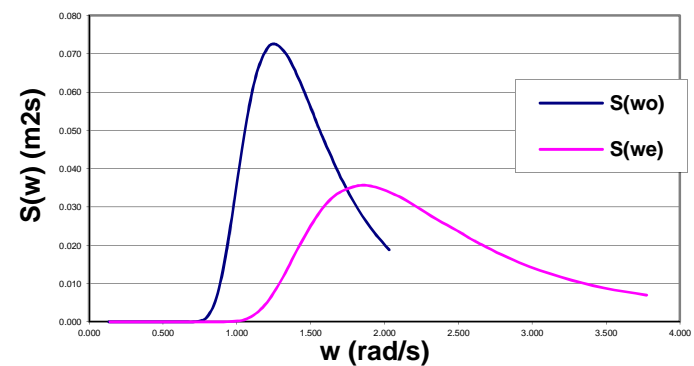

Fig. 6 Para un análisis realizado con un estado de mar 3, 8 nudos y 75\% de su desplazamiento.

A continuación se calcula el espectro de repuesta ec8, producto del cuadrado de la amplitud de respuesta por unidad de amplitud de ola incidente, multiplicado por el espectro de olas incidentes.

$$
\operatorname{Sw}(\omega)=\left(\mathrm{W}^{\wedge} 2\right) * \mathrm{~S} \eta(\omega)
$$

Siendo sus resultados mostrados en forma grafica en las figuras 7 y 8, para los movimientos del buque de HEAVE y PITCH respectivamente.

S-Heave(we)

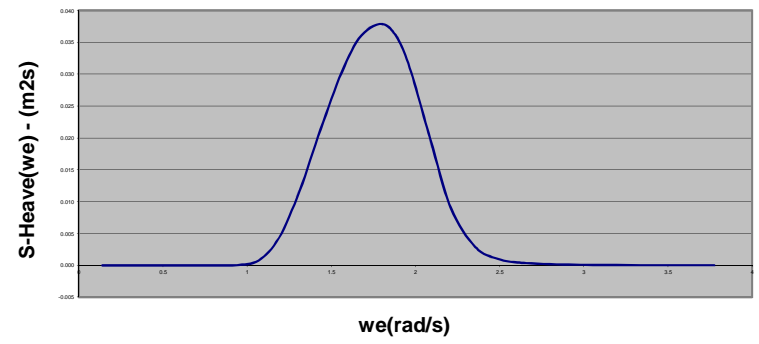

Fig. 7 Análisis realizado con un estado de mar 3, 8 nudos y $75 \%$ de su desplazamiento. 


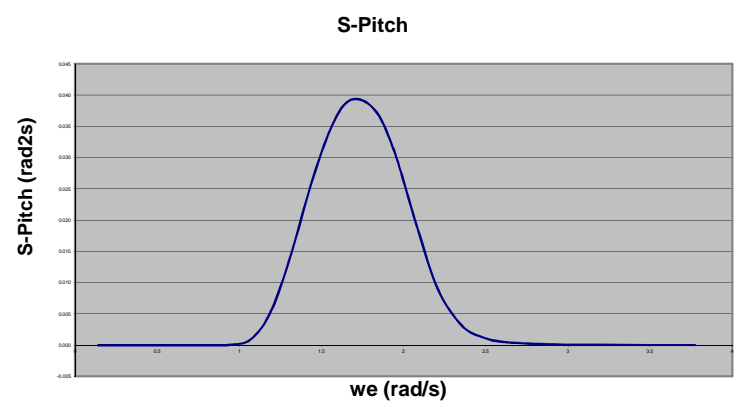

Fig. 8 Análisis realizado con un estado de mar 3, 8 nudos y $75 \%$ de su desplazamiento.

Como parte de nuestro cálculo se determinaran las áreas bajo las curvas de los espectros de respuestas que se puedan obtener, para ello se hace uso de la siguiente expresión:

$$
m_{n}=\oint^{\wedge} \mathrm{n} * \mathrm{~S}(\omega) * \mathrm{~d} \omega
$$

Donde:

$m_{n}=$ área de la curva del espectro de respuesta

\section{CALCULO DE LOS MOVIMIENTOS DEL BUQUE}

Las funciones que definen el movimiento del buque para los movimientos de heave y pitch, las cuales tienen como centro de coordenadas el centro de gravedad del buque y son desarrolladas para describir el movimiento vertical de un punto ubicado a una distancia $x_{i}$ del C.G., tienen la siguiente forma:

$$
\mathfrak{I}_{v i}=\mathfrak{I}-\chi_{i} \cdot \theta
$$

Heave: $\mathfrak{I}=\mathfrak{I}_{o} \cdot \cos \left(w_{e} \cdot t-\gamma_{\mathfrak{I}}\right)$

$$
\text { Pitch: } \theta=\theta_{o} \cdot \cos \left(w_{e} \cdot t-\gamma_{\theta}\right)
$$

De la suma de las funciones 10 y 11 obtenemos la función resultante que describe el movimiento vertical total del buque queda definido en ec12.

$$
\mathfrak{I}_{v}=\mathfrak{I}_{v o} \cdot \cos \left(w_{e} t-\gamma_{v}\right)
$$

Donde:

$$
\mathfrak{I}_{v o}=\sqrt{\mathfrak{I}_{o}^{2}+\left(x_{i} \cdot \theta_{o}\right)^{2}-2 \mathfrak{I}_{o} \cdot x_{i} \theta_{o} \cdot \cos \left(\gamma_{\mathfrak{I}}-\gamma_{\theta}\right)}
$$

$$
\gamma_{v}=\arctan \left[\frac{\mathfrak{I}_{o} \cdot \operatorname{sen} \gamma_{\mathfrak{I}}-x_{i} \cdot \theta_{o} \cdot \operatorname{sen} \gamma_{\theta}}{\mathfrak{I}_{o} \cdot \cos \gamma_{\mathfrak{I}}-x_{i} \cdot \theta_{o} \cdot \cos \gamma_{\theta}}\right]
$$

Así mismo la ecuación que define el movimiento relativo del buque queda definida con la siguiente expresión:

$$
\mathfrak{J}_{v R}=\mathfrak{I}_{v i}-h(x, t)
$$

Donde:

$\mathrm{h}=$ Ecuación que define el movimiento de la superficie del Mar.

$$
h=h_{a} \cdot \cos \left(k_{e} \cdot x_{i}-w_{e} \cdot t\right)
$$

$\mathfrak{I}_{v i}=$ Ecuación que define el movimiento del buque para un punto ubicado en $x_{i}$

$$
\mathfrak{I}_{v i}=\mathfrak{I}_{v o} \cdot \cos \left(w_{e} \cdot t-\gamma_{v}\right)
$$

de las ecuaciones 13 y 14 obtenemos la expresión representativa del movimiento relativo del buque Zvre.

$$
\mathfrak{J}_{\mathrm{vR}}=\mathfrak{J}_{\mathrm{vRo}} \cdot \cos \left(\mathrm{w}_{\mathrm{e}} \cdot \mathrm{t}-\gamma_{\mathrm{vR}}\right)
$$

Donde:

$$
\begin{gathered}
\mathfrak{I}_{v R o}=\sqrt{\mathfrak{I}_{v o}{ }^{2}+h_{a}{ }^{2}-2 \mathfrak{I}_{v o} \cdot h_{a} \cdot \cos \left(\gamma_{v}-k_{e} \cdot x_{i}\right)} \\
\gamma_{v R}=\arctan \left[\frac{\mathfrak{I}_{v o} \cdot \operatorname{sen} \gamma_{v}-h_{a} \cdot \operatorname{sen} k_{e} \cdot x_{i}}{\mathfrak{I}_{v o} \cdot \cos \gamma_{v}-h_{a} \cdot \cos k_{e} \cdot x_{i}}\right]
\end{gathered}
$$

\section{CALCULO DE LA PROBABILIDAD DE EMBARQUE DE AGUA}

Para determinar la probabilidad de embarque de agua ec 15 se debe determinar primero los valores de las áreas bajo las curvas de los espectros de movimiento $m_{o s}$ y la del área correspondiente al espectro de aceleración $m_{2 S}$ haciendo uso de ec.9.

$$
p\left\{\mathfrak{J}_{v R} \geq f^{\prime \prime}\right\}=e^{-\frac{\left(f^{\prime}\right)^{2}}{2 m_{o S}}}
$$


Donde:

$\mathrm{f}=$ francobordo

$m_{o S}=$ Área del espectro de movimiento relativo

Además se calcula el periodo característico de pitch con la siguiente fórmula:

$$
T \approx T_{\theta}=2 \pi \sqrt{\frac{2 m_{o S}}{2 m_{2 S}}}
$$

Por último para obtener el número de embarques de agua por hora se recurre a la siguiente relación:

$$
N_{T}=\frac{P}{T} \cdot 3600
$$

Donde:

$\mathrm{P}=$ Probabilidad de embarque

$\mathrm{T}=$ Periodo característico de pitch

\section{PROBABILIDAD DE EMERSIÓN DEL PROPULSOR}

La probabilidad de embarque de agua se calcula de la siguiente expresión:

$$
p\left\{\mathfrak{J}_{v R} \geq t\right\}=e^{-\frac{(t)^{2}}{2 m_{o S}}}
$$

Donde:

$\mathrm{t}=$ distancia del extremo superior del propulsor a la superficie del agua.

$m_{o S}=$ Área del espectro de movimiento relativo

\section{Aceleración vertical del buque}

Se determina la aceleración vertical del buque como resultado de la segunda derivada de la ecuación de movimiento del buque ec. 12 respecto del tiempo para una determinada posición a lo largo de la eslora del buque.

$$
\mathfrak{I}_{v}^{\prime \prime}=-w_{e}^{2} \cdot \mathfrak{I}_{v o} \cdot \cos \left(w_{e} t-\gamma_{v}\right)
$$

Donde:

$w_{e}=$ Frecuencia de encuentro

$\mathfrak{I}_{v o}=$ Amplitud del movimiento del buque

$\gamma_{v}=$ Angulo de desfasaje total

\section{Probabilidad de slamming}

La probabilidad de slamming es calculada de la suma de la probabilidad de emersión del propulsor ec16 y la probabilidad de que la amplitud de la velocidad relativa exceda la velocidad limite de slamming ec. 17 .

$$
p\left\{v \geq v_{0}\right\}=e^{-\frac{\left(v_{0}\right)^{2}}{2 m_{2 S}}}
$$

Donde:

$v_{\mathrm{o}}=$ Velocidad limita de Slamming

$m_{2 S}=$ Área bajo el espectro de velocidad relativa

La probabilidad de slammming resulta de la suma de las ecuaciones 16 y 17.

$p\{\operatorname{slam}\}=e^{-\left(\frac{\left(\mathrm{v}_{\mathrm{o}}\right)^{2}}{2 m_{2 S}}+\frac{(t)^{2}}{2 m_{o S}}\right)}$

\section{Probabilidad de degradación de la} performancia humana

Esta es la representación de la reducción del comfort que como consecuencia de su incremento dificulta el desarrollo de las labores de las personas abordo, siendo esta su ecuación representativa:

$$
S M=\left(3.087+1.392 .\left[\operatorname{Ln}\left(\frac{1}{2 . \pi} \sqrt{\frac{m_{2 S}}{m_{o S}}}\right)\right]^{2}\right) \cdot m_{4 S}{ }^{0.715}
$$

Donde:

$m_{o S}=$ Área del espectro de movimiento total

$m_{2 S}=$ Área bajo el espectro de velocidad total

$m_{4 S}=$ Área bajo el espectro de velocidad total

$S M=$ Magnitud subjetiva

\section{RESULTADOS}

Las tablas del 3 al 18 muestran los resultados de 
nuestros análisis, siguiendo el criterio mostrado en la Tabla 2. Así también la tabla19 muestra las probabilidades de degradación de la performancia humana para el mismo criterio.

Finalmente se muestra la tabla 20 con los resultados de la probabilidad de superar el criterio de aceleración para un punto de ubicación a $87.1 \%$ de la eslora.

\section{CONCLUSIONES}

Obtuvimos diversos espectros matemáticos como el espectro de movimiento buque, movimiento relativo del buque respecto a la superficie del mar, el espectro de respuestas del buque en heave y pitch de los cuales obtuvimos la información

necesaria para desarrollar el análisis propuesto y de esta manera determinar el Seakeeping del buque durante la etapa previa de diseño.

Podemos concluir que este buque pesquero presentará un comportamiento adecuado frente a los estados de mar más leves y estará en el límite de los criterios de diseño ante niveles superiores.

\section{REFERENCIAS}

Edwar Lewis, E., "Principles of Naval Architecture".

Rawson, K. J., "Basic Ship Theory".

Bhattacharyya, R., "Dynamics of Marine Vehicles".

Correspondencia: hegch16@yahoo.com.mx

\section{ANEXOS:}

Tabla 3. Análisis para estado de Mar 2, 8 nudos de velocidad y al 75\% de su desplazamiento.

\begin{tabular}{|l|l|r|r|r|r|r|c|}
\hline \multicolumn{1}{|c|}{ PROBABILIDADES } & \multicolumn{2}{|c|}{$\begin{array}{c}\text { Areas de } \\
\text { Espectros }\end{array}$} & $\begin{array}{c}\text { NO } \\
\text { EXCEDER }\end{array}$ & Unidades & $\begin{array}{c}\text { Criterios } \\
\text { Probabilidad } \\
\text { Limite }\end{array}$ & \multicolumn{1}{c|}{ Areas } & $\begin{array}{l}\text { Probabilidad } \\
\text { Calculada }\end{array}$ \\
\hline EMBARQUE DE AGUA & mo-zrel & 0.008 & 3.44 & $\mathrm{~m}$ & $5 \%$ & -783.87274 & $0.00 \%$ \\
\hline EMERSION DEL PROPULSOR & mo-Zprop & 0.009 & 0.187 & $\mathrm{~m}$ & $5 \%$ & -1.99992621 & $13.53 \%$ \\
\hline ACELERACIONES EN LAPROA & mo-actotal & 0.063 & 0.15 & $\mathrm{~g}$ & $5 \%$ & -17.1130444 & $0.00 \%$ \\
\hline SLAMMING & $\begin{array}{l}\text { mo-zrel } \\
\text { mo-vzrel }\end{array}$ & 0.008 & 3.44 & $\mathrm{~m}$ & $2 \%$ & -783.87274 & $0.00 \%$ \\
\hline
\end{tabular}

Tabla 4. Análisis para estado de Mar 2, 8 nudos de velocidad y al 100\% de su desplazamiento.

\begin{tabular}{|c|c|c|c|c|c|c|c|}
\hline PROBABILIDADES & \multicolumn{2}{|c|}{$\begin{array}{l}\text { Areas de } \\
\text { Espectros }\end{array}$} & $\begin{array}{c}\text { NO } \\
\text { EXCEDER }\end{array}$ & Unidades & \begin{tabular}{|c|} 
Criterios \\
Probabilidad \\
Limite
\end{tabular} & Areas & $\begin{array}{l}\text { Probabilidad } \\
\text { Calculada }\end{array}$ \\
\hline EMBARQUE DE AGUA & mo-zrel & 0.008 & 2.48 & $\mathrm{~m}$ & $5 \%$ & -392.146139 & $0.00 \%$ \\
\hline EMERSION DEL PROPULSOR & mo-Zprop & 0.008 & 1.147 & m(distancia & $5 \%$ & -77.4798275 & $0.00 \%$ \\
\hline ACELERACIONES EN LAPROA & mo-actotal & 0.008 & 0.15 & $g$ & $5 \%$ & -127.722226 & $0.00 \%$ \\
\hline SLAMMING & $\begin{array}{l}\text { mo-zrel } \\
\text { mo-vzrel }\end{array}$ & $\begin{array}{l}0.008 \\
0.101\end{array}$ & $\begin{array}{l}2.48 \\
1.61\end{array}$ & \begin{tabular}{|l|} 
m(calado) \\
mis(v. limite)
\end{tabular} & $2 \%$ & $\begin{array}{l}-392.146139 \\
-12.8412724\end{array}$ & $0.00 \%$ \\
\hline
\end{tabular}

Tabla 5. Análisis para estado de Mar 2, 11 nudos de velocidad y al 75\% de su desplazamiento. 


\begin{tabular}{|c|c|c|c|c|c|c|c|}
\hline PROBABILIDADES & \multicolumn{2}{|c|}{$\begin{array}{l}\text { Areas de } \\
\text { Espectros }\end{array}$} & $\begin{array}{c}\text { NO } \\
\text { EXCEDER }\end{array}$ & Unidades & \begin{tabular}{|c|} 
Criterios \\
Probabilidad \\
Limite
\end{tabular} & Areas & $\begin{array}{l}\text { Probabilidad } \\
\text { Calculada }\end{array}$ \\
\hline EMBARQUE DE AGUA & mo-zrel & 0.006 & 3.44 & $\mathrm{~m}$ & $5 \%$ & -1003.28516 & $0.00 \%$ \\
\hline EMERSIONN DEL PROPULSOR & mo-Zprop & 0.007 & 0.187 & m(distancia & $5 \%$ & -2.58422485 & $7.55 \%$ \\
\hline ACELERACIONES EN LA PROA & mo-actotal & 0.055 & 0.15 & g & $5 \%$ & -19.8364469 & $0.00 \%$ \\
\hline SLAMMING & $\begin{array}{l}\text { mo-zrel } \\
\text { mo-vzrel }\end{array}$ & $\begin{array}{l}0.006 \\
0.064\end{array}$ & $\begin{array}{l}3.44 \\
1.49\end{array}$ & $\begin{array}{l}\text { m(calado) } \\
\text { m/s(v. limite) }\end{array}$ & $2 \%$ & $\begin{array}{l}-1003.28516 \\
-17.4233573\end{array}$ & $0.00 \%$ \\
\hline
\end{tabular}

Tabla 6. Análisis para estado de Mar 2, 11 nudos de velocidad y al 100\% de su desplazamiento.

\begin{tabular}{|c|c|c|c|c|c|c|c|}
\hline PROBABILIDADES & \multicolumn{2}{|c|}{$\begin{array}{c}\text { Areas de } \\
\text { Espectros }\end{array}$} & $\begin{array}{c}\text { NO } \\
\text { EXCEDER }\end{array}$ & Unidades & \begin{tabular}{|c|} 
Criterios \\
Probabilidad \\
Limite \\
\end{tabular} & Areas & $\begin{array}{l}\text { Probabilidad } \\
\text { Calculada }\end{array}$ \\
\hline EMBARQUE DE AGUA & mo-zrel & 0.008 & 2.48 & $m$ & $5 \%$ & -405.545871 & $0.00 \%$ \\
\hline EMERSION DEL PROPULSOR & mo-Zprop & 0.008 & 1.147 & m(distancia & $5 \%$ & -80.6792749 & $0.00 \%$ \\
\hline ACELERACIONES EN LAPROA & mo-actotal & 0.009 & 0.15 & $g$ & $5 \%$ & -125.680921 & $0.00 \%$ \\
\hline SLAMMING & $\begin{array}{l}\text { mo-zrel } \\
\text { mo-vzrel }\end{array}$ & $\begin{array}{l}0.008 \\
0.138\end{array}$ & $\begin{array}{l}2.48 \\
1.61\end{array}$ & $\begin{array}{l}\text { m(calado) } \\
\text { mis(v. limite) }\end{array}$ & $2 \%$ & $\begin{array}{l}-405.545871 \\
-9.41003785\end{array}$ & $0.00 \%$ \\
\hline
\end{tabular}

Tabla 7. Análisis para estado de Mar 3, 8 nudos de velocidad y al 75\% de su desplazamiento.

\begin{tabular}{|c|c|c|c|c|c|c|c|}
\hline PROBABILIDADES & \multicolumn{2}{|c|}{$\begin{array}{l}\text { Areas de } \\
\text { Espectros }\end{array}$} & $\begin{array}{c}\text { NO } \\
\text { EXCEDER }\end{array}$ & Unidades & \begin{tabular}{|c|} 
Criterios \\
Probabilidad \\
Limite
\end{tabular} & Areas & $\begin{array}{l}\text { Probabilidad } \\
\text { Calculada }\end{array}$ \\
\hline EMBARQUE DE AGUA & mo-zrel & 0.345 & 3.44 & $m$ & $5 \%$ & -17.143282 & $0.00 \%$ \\
\hline EMERSIÓN DEL PROPULSOR & mo-Zprop & 0.250 & 0.187 & m(distancia & $5 \%$ & -0.06989502 & $93.25 \%$ \\
\hline ACELERACIONES EN LA PROA & mo-actotal & 2.413 & 0.15 & $g$ & $5 \%$ & -0.44869165 & $63.85 \%$ \\
\hline SLAMMING & $\begin{array}{l}\text { mo-zrel } \\
\text { mo-vzrel }\end{array}$ & $\begin{array}{l}0.345 \\
1.362\end{array}$ & $\begin{array}{l}3.44 \\
1.49\end{array}$ & $\begin{array}{l}\text { m(calado) } \\
\text { m/s(v. limite) }\end{array}$ & $2 \%$ & $\begin{array}{r}-17.143282 \\
-0.81610426\end{array}$ & $0.00 \%$ \\
\hline
\end{tabular}

Tabla 8. Análisis para estado de Mar 3, 8 nudos de velocidad y al 100\% de su desplazamiento.

\begin{tabular}{|c|c|c|c|c|c|c|c|}
\hline PROBABILIDADES & \multicolumn{2}{|c|}{$\begin{array}{l}\text { Areas de } \\
\text { Espectros }\end{array}$} & $\begin{array}{c}\text { NO } \\
\text { EXCEDER }\end{array}$ & Unidades & \begin{tabular}{|c|}
$\begin{array}{c}\text { Criterios } \\
\text { Probabilidad } \\
\text { Limite }\end{array}$ \\
\end{tabular} & Areas & $\begin{array}{l}\text { Probabilidad } \\
\text { Calculada } \\
\end{array}$ \\
\hline EMBARQUE DE AGUA & mo-zrel & 0.274 & 2.48 & $m$ & $5 \%$ & -11.2283372 & $0.00 \%$ \\
\hline EMERSIÓN DEL PROPULSOR & mo-Zprop & 0.202 & 1.147 & m(distancia & $5 \%$ & -3.25749424 & $3.85 \%$ \\
\hline ACELERACIONES EN LA PROA & mo-actotal & 1.105 & 0.15 & g & $5 \%$ & -0.97971562 & $37.54 \%$ \\
\hline SLAMMING & $\begin{array}{l}\text { mo-zrel } \\
\text { mo-vzrel }\end{array}$ & $\begin{array}{l}0.274 \\
1.029\end{array}$ & $\begin{array}{l}2.48 \\
1.61\end{array}$ & $\begin{array}{l}\text { m(calado) } \\
\text { mis(v. limite) }\end{array}$ & $2 \%$ & $\begin{array}{l}-11.2283372 \\
-1.26212814\end{array}$ & $0.00 \%$ \\
\hline
\end{tabular}

Tabla 9. Análisis para estado de Mar 3, 11 nudos de velocidad y al 75\% de su desplazamiento. 


\begin{tabular}{|c|c|c|c|c|c|c|c|}
\hline PROBABILIDADES & \multicolumn{2}{|c|}{$\begin{array}{l}\text { Areas de } \\
\text { Espectros }\end{array}$} & $\begin{array}{c}\text { NO } \\
\text { EXCEDER }\end{array}$ & Unidades & \begin{tabular}{|c|} 
Criterios \\
Probabilidad \\
Limite
\end{tabular} & Areas & $\begin{array}{l}\text { Probabilidad } \\
\text { Calculada }\end{array}$ \\
\hline EMBARQUE DE AGUA & mo-zrel & 0.307 & 3.44 & $m$ & $5 \%$ & -19.28099 & $0.00 \%$ \\
\hline EMERSIÓN DEL PROPULSOR & mo-Zprop & 0.218 & 0.187 & m(distancia & $5 \%$ & -0.08011343 & $92.30 \%$ \\
\hline ACELERACIONES EN LA PROA & mo-actotal & 2.908 & 0.15 & $g$ & $5 \%$ & -0.37235224 & $68.91 \%$ \\
\hline SLAMMING & \begin{tabular}{|l|} 
mo-zrel \\
mo-vzrel
\end{tabular} & $\begin{array}{l}0.307 \\
1.467\end{array}$ & $\begin{array}{l}3.44 \\
1.49\end{array}$ & $\begin{array}{l}\text { m(calado) } \\
\text { m/s(v. limite })\end{array}$ & $2 \%$ & $\begin{array}{r}-19.28099 \\
-0.75760581\end{array}$ & $0.00 \%$ \\
\hline
\end{tabular}

Tabla10. Análisis para estado de Mar 3, 11 nudos de velocidad y al 100\% de su desplazamiento.

\begin{tabular}{|c|c|c|c|c|c|c|c|}
\hline PROBABILIDADES & \multicolumn{2}{|c|}{$\begin{array}{l}\text { Areas de } \\
\text { Espectros }\end{array}$} & $\begin{array}{c}\text { NO } \\
\text { EXCEDER }\end{array}$ & Unidades & $\begin{array}{c}\text { Criterios } \\
\text { Probabilidad } \\
\text { Limite }\end{array}$ & Areas & $\begin{array}{l}\text { Probabilidad } \\
\text { Calculada }\end{array}$ \\
\hline EMBARQUE DE AGUA & mo-zrel & 0.235 & 2.48 & $m$ & $5 \%$ & -13.0629562 & $0.00 \%$ \\
\hline EMERSIÓN DEL PROPULSOR & mo-Zprop & 0.153 & 1.147 & m(distancia & $5 \%$ & -4.29145978 & $1.37 \%$ \\
\hline ACELERACIONES EN LAPROA & mo-actotal & 1.166 & 0.15 & $g$ & $5 \%$ & -0.92888896 & $39.50 \%$ \\
\hline SLAMMING & $\begin{array}{l}\text { mo-zrel } \\
\text { mo-vzrel }\end{array}$ & $\begin{array}{l}0.235 \\
1.123\end{array}$ & $\begin{array}{l}2.48 \\
1.61\end{array}$ & $\begin{array}{l}\text { m(calado) } \\
\text { m/s(v. limite) }\end{array}$ & $2 \%$ & $\begin{array}{l}-13.0629562 \\
-1.15602508\end{array}$ & $0.00 \%$ \\
\hline
\end{tabular}

Tabla 11. Análisis para estado de Mar 4, 8 nudos de velocidad y al 75\% de su desplazamiento.

\begin{tabular}{|c|c|c|c|c|c|c|c|}
\hline PROBABILIDADES & \multicolumn{2}{|c|}{$\begin{array}{l}\text { Areas de } \\
\text { Espectros }\end{array}$} & $\begin{array}{c}\text { NO } \\
\text { EXCEDER }\end{array}$ & Unidades & $\begin{array}{c}\text { Criterios } \\
\text { Probabilidad } \\
\text { Limite }\end{array}$ & Areas & $\begin{array}{l}\text { Probabilidad } \\
\text { Calculada }\end{array}$ \\
\hline EMBARQUE DE AGUA & mo-zrel & 1.300 & 3.44 & $\mathrm{~m}$ & $5 \%$ & -4.54983038 & $1.06 \%$ \\
\hline EMERSION DEL PROPULSOR & mo-Zprop & 0.660 & 0.187 & m(distancia & $5 \%$ & -0.02650373 & $97.38 \%$ \\
\hline ACELERACIONES EN LAPROA & mo-actotal & 6.370 & 0.15 & $g$ & $5 \%$ & -0.1699522 & $84.37 \%$ \\
\hline SLAMMING & $\begin{array}{l}\text { mo-zrel } \\
\text { mo-vzrel }\end{array}$ & \begin{tabular}{l|}
1.300 \\
3.804
\end{tabular} & $\begin{array}{l}3.44 \\
1.49\end{array}$ & \begin{tabular}{|l|}
$\mathrm{m}(\mathrm{calado})$ \\
$\mathrm{m} / \mathrm{s}(\mathrm{v}$. limite $)$
\end{tabular} & $2 \%$ & $\begin{array}{l}-4.54983038 \\
-0.29215656\end{array}$ & $0.79 \%$ \\
\hline
\end{tabular}

Tabla 12. Análisis para estado de Mar 4, 8 nudos de velocidad y al 100\% de su desplazamiento.

\begin{tabular}{|c|c|c|c|c|c|c|c|}
\hline PROBABILIDADES & \multicolumn{2}{|c|}{$\begin{array}{l}\text { Areas de } \\
\text { Espectros }\end{array}$} & $\begin{array}{c}\text { NO } \\
\text { EXCEDER }\end{array}$ & Unidades & \begin{tabular}{|c|} 
Criterios \\
Probabilidad \\
Limite
\end{tabular} & Areas & $\begin{array}{l}\text { Probabilidad } \\
\text { Calculada }\end{array}$ \\
\hline EMBARQUE DE AGUA & mo-zrel & 1.383 & 2.48 & $m$ & $5 \%$ & -2.22353045 & $10.82 \%$ \\
\hline EMERSION DEL PROPULSOR & mo-Zprop & 0.797 & 1.147 & m(distancia & $5 \%$ & -0.82518411 & $43.82 \%$ \\
\hline ACELERACIONES EN LA PROA & mo-actotal & 4.562 & 0.15 & $g$ & $5 \%$ & -0.23734175 & $78.87 \%$ \\
\hline SLAMMING & $\begin{array}{l}\text { mo-zrel } \\
\text { mo-vzrel }\end{array}$ & $\begin{array}{l}1.383 \\
3.512 \\
\end{array}$ & $\begin{array}{l}2.48 \\
1.61 \\
\end{array}$ & $\begin{array}{l}\text { m(calado) } \\
\text { m/s(v. limite) }\end{array}$ & $2 \%$ & \begin{tabular}{|l|}
-2.22353045 \\
-0.36958891 \\
\end{tabular} & $7.48 \%$ \\
\hline
\end{tabular}

Tabla 13. Análisis para estado de Mar 4, 11 nudos de velocidad y al 75\% de su desplazamiento. 


\begin{tabular}{|c|c|c|c|c|c|c|c|}
\hline PROBABILIDADES & \multicolumn{2}{|c|}{$\begin{array}{l}\text { Areas de } \\
\text { Espectros }\end{array}$} & $\begin{array}{c}\text { NO } \\
\text { EXCEDER }\end{array}$ & Unidades & \begin{tabular}{|c|} 
Criterios \\
Probabilidad \\
Limite
\end{tabular} & Areas & $\begin{array}{l}\text { Probabilidad } \\
\text { Calculada }\end{array}$ \\
\hline EMBARQUE DE AGUA & mo-zrel & 1.278 & 3.44 & m & $5 \%$ & -4.63021174 & $0.98 \%$ \\
\hline EMERSIÓN DEL PROPULSOR & mo-Zprop & 0.687 & 0.187 & m(distancia & $5 \%$ & -0.02544619 & $97.49 \%$ \\
\hline ACELERACIONES EN LA PROA & mo-actotal & 8.988 & 0.15 & $g$ & $5 \%$ & -0.12045215 & $88.65 \%$ \\
\hline SLAMMING & $\begin{array}{l}\text { mo-zrel } \\
\text { mo-vzrel }\end{array}$ & $\begin{array}{l}1.278 \\
4.443\end{array}$ & $\begin{array}{l}3.44 \\
1.49\end{array}$ & $\begin{array}{l}\text { m(calado) } \\
\text { mis(v. limite) }\end{array}$ & $2 \%$ & $\begin{array}{r}-4.63021174 \\
-0.2501405\end{array}$ & $0.76 \%$ \\
\hline
\end{tabular}

Tabla 14. Análisis para estado de Mar 4, 11 nudos de velocidad y al 100\% de su desplazamiento.

\begin{tabular}{|c|c|c|c|c|c|c|c|}
\hline PROBABILIDADES & \multicolumn{2}{|c|}{$\begin{array}{l}\text { Areas de } \\
\text { Espectros }\end{array}$} & $\begin{array}{c}\text { NO } \\
\text { EXCEDER }\end{array}$ & Unidades & $\begin{array}{c}\text { Criterios } \\
\text { Probabilidad } \\
\text { Limite }\end{array}$ & Areas & $\begin{array}{l}\text { Probabilidad } \\
\text { Calculada }\end{array}$ \\
\hline EMBARQUE DE AGUA & mo-zrel & 1.376 & 2.48 & $m$ & $5 \%$ & -2.23530903 & $10.70 \%$ \\
\hline EMERSIÓN DEL PROPULSOR & mo-Zprop & 0.777 & 1.147 & m(distancia & $5 \%$ & -0.84713269 & $42.86 \%$ \\
\hline ACELERACIONES EN LAPROA & mo-actotal & 6.174 & 0.15 & g & $5 \%$ & -0.1753446 & $83.92 \%$ \\
\hline SLAMMING & $\begin{array}{l}\text { mo-zrel } \\
\text { mo-vzrel }\end{array}$ & $\begin{array}{l}1.376 \\
4.127\end{array}$ & $\begin{array}{l}2.48 \\
1.61\end{array}$ & $\begin{array}{l}\text { m(calado) } \\
\text { mis(v. limite) }\end{array}$ & $2 \%$ & $\begin{array}{l}-2.23530903 \\
-0.31455644\end{array}$ & $7.81 \%$ \\
\hline
\end{tabular}

Tabla 15. Análisis para estado de Mar 5, 8 nudos de velocidad y al 75\% de su desplazamiento.

\begin{tabular}{|c|c|c|c|c|c|c|c|}
\hline PROBABILIDADES & \multicolumn{2}{|c|}{$\begin{array}{l}\text { Areas de } \\
\text { Espectros }\end{array}$} & $\begin{array}{c}\text { NO } \\
\text { EXCEDER }\end{array}$ & Unidades & \begin{tabular}{|c|} 
Criterios \\
$\begin{array}{c}\text { Probabilidad } \\
\text { Limite }\end{array}$
\end{tabular} & Areas & $\begin{array}{l}\text { Probabilidad } \\
\text { Calculada }\end{array}$ \\
\hline EMBARQUE DE AGUA & mo-zrel & 1.898 & 3.44 & $m$ & $5 \%$ & -3.11730375 & $4.43 \%$ \\
\hline EMERSIÓN DEL PROPULSOR & mo-Zprop & 0.820 & 0.187 & m(distancia & $5 \%$ & -0.02133174 & $97.89 \%$ \\
\hline ACELERACIONES EN LA PROA & mo-actotal & 7.920 & 0.15 & $g$ & $5 \%$ & -0.13669069 & $87.22 \%$ \\
\hline SLAMMING & $\begin{array}{l}\text { mo-zrel } \\
\text { mo-vzrel }\end{array}$ & $\begin{array}{l}1.898 \\
4.928\end{array}$ & $\begin{array}{l}3.44 \\
1.49\end{array}$ & $\begin{array}{l}\text { m(calado) } \\
\text { m/s(v. limite) }\end{array}$ & $2 \%$ & $\begin{array}{l}-3.11730375 \\
-0.22553792\end{array}$ & $3.53 \%$ \\
\hline
\end{tabular}

Tabla 16. Análisis para estado de Mar 5, 8 nudos de velocidad y al 100\% de su desplazamiento.

\begin{tabular}{|c|c|c|c|c|c|c|c|}
\hline PROBABILIDADES & \multicolumn{2}{|c|}{$\begin{array}{l}\text { Areas de } \\
\text { Espectros }\end{array}$} & $\begin{array}{c}\text { NO } \\
\text { EXCEDER }\end{array}$ & Unidades & \begin{tabular}{c|} 
Criterios \\
Probabilidad \\
Limite
\end{tabular} & Areas & $\begin{array}{l}\text { Probabilidad } \\
\text { Calculada }\end{array}$ \\
\hline EMBARQUE DE AGUA & mo-zrel & 2.146 & 2.48 & $m$ & $5 \%$ & -1.43312287 & $23.86 \%$ \\
\hline EMERSIÓN DEL PROPULSOR & mo-Zprop & 1.088 & 1.147 & m(distancia & $5 \%$ & -0.60484797 & $54.62 \%$ \\
\hline ACELERACIONES EN LA PROA & mo-actotal & 6.207 & 0.15 & $g$ & $5 \%$ & -0.17441155 & $84.00 \%$ \\
\hline SLAMMING & $\begin{array}{l}\text { mo-zrel } \\
\text { mo-vzrel }\end{array}$ & $\begin{array}{l}2.146 \\
4.833 \\
\end{array}$ & $\begin{array}{l}2.48 \\
1.61 \\
\end{array}$ & \begin{tabular}{|l|} 
m(calado) \\
m/s(v. limite)
\end{tabular} & $2 \%$ & $\begin{array}{l}-1.43312287 \\
-0.26860367\end{array}$ & $18.24 \%$ \\
\hline
\end{tabular}

Tabla 17. Análisis para estado de Mar 5, 11 nudos de velocidad y al 75\% de su desplazamiento. 


\begin{tabular}{|c|c|c|c|c|c|c|c|}
\hline PROBABILIDADES & \multicolumn{2}{|c|}{$\begin{array}{l}\text { Areas de } \\
\text { Espectros }\end{array}$} & $\begin{array}{c}\text { NO } \\
\text { EXCEDER }\end{array}$ & Unidades & $\begin{array}{c}\text { Criterios } \\
\text { Probabilidad } \\
\text { Limite }\end{array}$ & Areas & $\begin{array}{l}\text { Probabilidad } \\
\text { Calculada }\end{array}$ \\
\hline EMBARQUE DE AGUA & mo-zrel & 1.898 & 3.44 & $m$ & $5 \%$ & -3.1173383 & $4.43 \%$ \\
\hline EMERSION DEL PROPULSOR & mo-Zprop & 0.886 & 0.187 & m(distancia & $5 \%$ & -0.01973151 & $98.05 \%$ \\
\hline ACELERACIONES EN LA PROA & mo-actotal & 11.501 & 0.15 & $g$ & $5 \%$ & -0.09413549 & $91.02 \%$ \\
\hline SLAMMING & $\begin{array}{l}\text { mo-zrel } \\
\text { mo-vzrel }\end{array}$ & $\begin{array}{l}1.898 \\
5.852\end{array}$ & $\begin{array}{l}3.44 \\
1.49\end{array}$ & $\begin{array}{l}\text { m(calado) } \\
\text { m/s(v. limite) }\end{array}$ & $2 \%$ & $\begin{array}{r}-3.1173383 \\
-0.18994478 \\
\end{array}$ & $3.66 \%$ \\
\hline
\end{tabular}

Tabla 18. Análisis para estado de Mar 5, 11 nudos de velocidad y al 100\% de su desplazamiento.

\begin{tabular}{|c|c|c|c|c|c|c|c|}
\hline PROBABILIDADES & \multicolumn{2}{|c|}{$\begin{array}{l}\text { Areas de } \\
\text { Espectros }\end{array}$} & $\begin{array}{c}\text { NO } \\
\text { EXCEDER }\end{array}$ & Unidades & $\begin{array}{c}\text { Criterios } \\
\text { Probabilidad } \\
\text { Limite }\end{array}$ & Areas & $\begin{array}{l}\text { Probabilidad } \\
\text { Calculada }\end{array}$ \\
\hline EMBARQUE DE AGUA & mo-zrel & 2.189 & 2.48 & $m$ & $5 \%$ & -1.40499807 & $24.54 \%$ \\
\hline EMERSION DEL PROPULSOR & mo-Zprop & 1.125 & 1.147 & m(distancia & $5 \%$ & -0.58485628 & $55.72 \%$ \\
\hline ACELERACIONES EN LAPROA & mo-actotal & 8.802 & 0.15 & g & $5 \%$ & -0.12300612 & $88.43 \%$ \\
\hline SLAMMING & $\begin{array}{l}\text { mo-zrel } \\
\text { mo-vrel }\end{array}$ & $\begin{array}{l}2.189 \\
5.804\end{array}$ & $\begin{array}{l}2.48 \\
1.61 \\
\end{array}$ & $\begin{array}{l}\text { m(calado) } \\
\text { mis(v. limite) }\end{array}$ & $2 \%$ & $\begin{array}{l}-1.40499807 \\
-0.22366766 \\
\end{array}$ & $19.62 \%$ \\
\hline
\end{tabular}

Tabla 19. Resultados de las Probabilidades de degradación de la performancia humana.

\begin{tabular}{|c|c|c|c|c|c|}
\hline & & Desplazamiento & $\begin{array}{l}\text { Magnitud } \\
\text { Subjectiva }\end{array}$ & $\begin{array}{c}\text { Criterio } \\
\text { Limite }\end{array}$ & $\begin{array}{l}\text { Probabilidac } \\
\text { Calculada }\end{array}$ \\
\hline \multirow{4}{*}{$\begin{array}{c}\text { Tipo de Mar } \\
2\end{array}$} & \multirow{2}{*}{$\begin{array}{l}\text { Velocidad } \\
8 \text { nudos }\end{array}$} & \multirow{2}{*}{\begin{tabular}{|r|}
$75 \%$ \\
$100 \%$ \\
\end{tabular}} & 0.13 & 10 & 1.3 \\
\hline & & & 0.14 & 10 & 1.4 \\
\hline & \multirow{2}{*}{$\begin{array}{l}\text { Velocidad } \\
11 \text { nudos }\end{array}$} & \multirow{2}{*}{$\begin{array}{c}75 \% \\
100 \% \\
\end{array}$} & 0.10 & 10 & 1.0 \\
\hline & & & 0.12 & 10 & 1.2 \\
\hline \multirow{4}{*}{$\begin{array}{c}\text { Tipo de Mar } \\
3\end{array}$} & \multirow{2}{*}{$\begin{array}{c}\text { Velocidad } \\
8 \text { nudos }\end{array}$} & \multirow{2}{*}{$\begin{array}{c}75 \% \\
100 \%\end{array}$} & 2.40 & 10 & 24.0 \\
\hline & & & 2.20 & 10 & 22.0 \\
\hline & \multirow{2}{*}{$\begin{array}{l}\text { Velocidad } \\
11 \text { nudos }\end{array}$} & \multirow{2}{*}{$\begin{array}{c}75 \% \\
100 \%\end{array}$} & 2.11 & 10 & 21.1 \\
\hline & & & 1.90 & 10 & 19.0 \\
\hline \multirow{4}{*}{$\begin{array}{c}\text { Tipo de Mar } \\
4\end{array}$} & \multirow{2}{*}{$\begin{array}{c}\text { Velocidad } \\
8 \text { nudos }\end{array}$} & \multirow{2}{*}{$\begin{array}{c}75 \% \\
100 \%\end{array}$} & 6.77 & 10 & 67.7 \\
\hline & & & 7.54 & 10 & 75.4 \\
\hline & \multirow{2}{*}{$\begin{array}{l}\text { Velocidad } \\
11 \text { nudos }\end{array}$} & \multirow{2}{*}{$\begin{array}{l}75 \% \\
100 \%\end{array}$} & 6.37 & 10 & 63.7 \\
\hline & & & 7.21 & 10 & 72.1 \\
\hline \multirow{4}{*}{$\begin{array}{c}\text { Tipo de Mar } \\
5\end{array}$} & \multirow{2}{*}{$\begin{array}{l}\text { Velocidad } \\
8 \text { nudos }\end{array}$} & \multirow{2}{*}{$\begin{array}{c}75 \% \\
100 \%\end{array}$} & 9.28 & 10 & 92.8 \\
\hline & & & 10.69 & 10 & 106.9 \\
\hline & \multirow{2}{*}{$\begin{array}{l}\text { Velocidad } \\
11 \text { nudos }\end{array}$} & \multirow{2}{*}{$\begin{array}{c}75 \% \\
100 \%\end{array}$} & 8.81 & 10 & 88.1 \\
\hline & & & 10.36 & 10 & 103.6 \\
\hline
\end{tabular}

Tabla 20. Resultados de Probabilidad para superar el criterio de aceleración a $87.1 \%$ de la Eslora total. 


\begin{tabular}{|c|c|c|c|c|c|c|c|c|c|}
\hline \\
\hline & & Desplazamiento & \multicolumn{2}{|c|}{$\begin{array}{l}\text { Areas de } \\
\text { Espectros }\end{array}$} & \multirow{2}{*}{$\begin{array}{c}\text { NO } \\
\text { EXCEDER }\end{array}$} & \multirow{2}{*}{$\begin{array}{c}\text { Unidades } \\
\mathrm{g}\end{array}$} & \multirow{2}{*}{$\begin{array}{c}\begin{array}{c}\text { Criterios } \\
\text { Probabilidad } \\
\text { Limite }\end{array} \\
5 \% \\
\end{array}$} & \multirow{2}{*}{$\frac{\text { Areas }}{-20.528}$} & \multirow{2}{*}{$\begin{array}{l}\text { Probabilidad } \\
\text { Calculada (\%) }\end{array}$} \\
\hline \multirow{4}{*}{$\begin{array}{c}\text { Tipo de Mar } \\
2\end{array}$} & \multirow{2}{*}{$\begin{array}{c}\text { Velocidad } \\
8 \text { nudos }\end{array}$} & \multirow{2}{*}{$\begin{array}{l}75 \% \\
100 \%\end{array}$} & mo-actotal & 0.053 & & & & & \\
\hline & & & mo-actotal & 0.007 & 0.15 & $\mathrm{~g}$ & $5 \%$ & -163.333 & $0 \%$ \\
\hline & Velocidad & $75 \%$ & mo-actotal & 0.046 & 0.15 & $\mathrm{~g}$ & $5 \%$ & -23.764 & $0 \%$ \\
\hline & 11 nudos & $100 \%$ & mo-actotal & 0.0066 & 0.15 & $\mathrm{~g}$ & $5 \%$ & -163.591 & $0 \%$ \\
\hline \multirow{4}{*}{$\begin{array}{c}\text { Tipo de Mar } \\
3\end{array}$} & \multirow{2}{*}{$\begin{array}{c}\text { Velocidad } \\
8 \text { nudos }\end{array}$} & \multirow{2}{*}{$\begin{array}{l}75 \% \\
100 \%\end{array}$} & mo-actotal & 2.0373 & 0.15 & $\mathrm{~g}$ & $5 \%$ & -0.531 & $59 \%$ \\
\hline & & & mo-actotal & 0.9002 & 0.15 & $\mathrm{~g}$ & $5 \%$ & -1.203 & $30 \%$ \\
\hline & \multirow{2}{*}{$\begin{array}{l}\text { Velocidad } \\
11 \text { nudos }\end{array}$} & \multirow{2}{*}{$\begin{array}{l}75 \% \\
100 \%\end{array}$} & mo-actotal & 2.4701 & 0.15 & $g$ & $5 \%$ & -0.438 & $65 \%$ \\
\hline & & & mo-actotal & 0.9539 & 0.15 & g & $5 \%$ & -1.135 & $32 \%$ \\
\hline \multirow{4}{*}{$\begin{array}{c}\text { Tipo de Mar } \\
4\end{array}$} & \multirow{2}{*}{$\begin{array}{l}\text { Velocidad } \\
8 \text { nudos }\end{array}$} & \multirow{2}{*}{$\begin{array}{l}75 \% \\
100 \%\end{array}$} & mo-actotal & 5.4133 & 0.15 & $\mathrm{~g}$ & $5 \%$ & -0.200 & $82 \%$ \\
\hline & & & mo-actotal & 3.7598 & 0.15 & $\mathrm{~g}$ & $5 \%$ & -0.288 & $75 \%$ \\
\hline & \multirow{2}{*}{$\begin{array}{l}\text { Velocidad } \\
11 \text { nudos }\end{array}$} & \multirow{2}{*}{$\begin{array}{l}75 \% \\
100 \%\end{array}$} & mo-actotal & 7.6902 & 0.15 & $\mathrm{~g}$ & $5 \%$ & -0.141 & $87 \%$ \\
\hline & & & mo-actotal & 5.125 & 0.15 & $\mathrm{~g}$ & $5 \%$ & -0.211 & $81 \%$ \\
\hline \multirow{4}{*}{$\begin{array}{c}\text { Tipo de Mar } \\
5\end{array}$} & \multirow{2}{*}{$\begin{array}{l}\text { Velocidad } \\
8 \text { nudos }\end{array}$} & \multirow{2}{*}{$\begin{array}{l}75 \% \\
100 \%\end{array}$} & mo-actotal & 6.749 & 0.15 & $\mathrm{~g}$ & $5 \%$ & -0.160 & $85 \%$ \\
\hline & & & mo-actotal & 5.136 & 0.15 & $\mathrm{~g}$ & $5 \%$ & -0.211 & $81 \%$ \\
\hline & \multirow{2}{*}{$\begin{array}{l}\text { Velocidad } \\
11 \text { nudos }\end{array}$} & \multirow{2}{*}{$\begin{array}{l}75 \% \\
100 \%\end{array}$} & mo-actotal & 11.501 & 0.15 & $\mathrm{~g}$ & $5 \%$ & -0.094 & $91 \%$ \\
\hline & & & mo-actotal & 7.3355 & 0.15 & g & $5 \%$ & -0.148 & $86 \%$ \\
\hline
\end{tabular}

